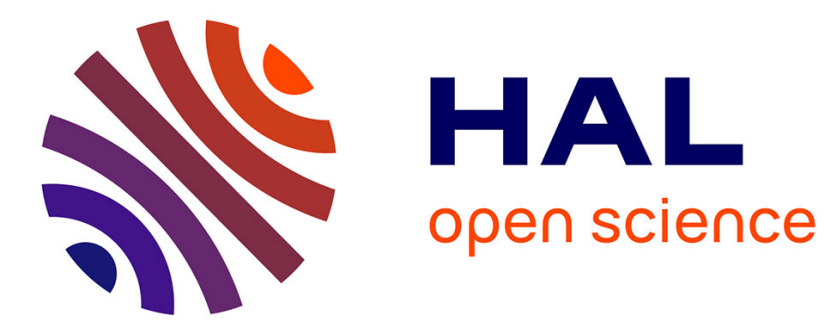

\title{
NUMERICAL MODELLING OF CVD PROCESSES AND EQUIPMENT
}

\author{
C. Werner
}

\section{To cite this version:}

C . Werner. NUMERICAL MODELLING OF CVD PROCESSES AND EQUIPMENT. Journal de Physique IV Proceedings, 1991, 02 (C2), pp.C2-3-C2-18. 10.1051/jp4:1991201 • jpa-00249768

\section{HAL Id: jpa-00249768 https://hal.science/jpa-00249768}

Submitted on 1 Jan 1991

HAL is a multi-disciplinary open access archive for the deposit and dissemination of scientific research documents, whether they are published or not. The documents may come from teaching and research institutions in France or abroad, or from public or private research centers.
L'archive ouverte pluridisciplinaire HAL, est destinée au dépôt et à la diffusion de documents scientifiques de niveau recherche, publiés ou non, émanant des établissements d'enseignement et de recherche français ou étrangers, des laboratoires publics ou privés. 
Colloque C2, suppl. au Journal de Physique II, Vol 1, septembre 1991

\title{
NUMERICAL MODELLING OF CVD PROCESSES AND EOUIPMENT
}

\author{
C. WERNER \\ SIEMENS AG, ZFE SPT 33, Otto-Hahn-Ring 6, D-8000 Munich 83, \\ Germany
}

\begin{abstract}
A review is given on actual numerical models for CVD equipment and processes. While the basic work concerning the differential equations and the boundary conditions has been established already a decade or more ago, the more recent availability of increased computing power has enabled simulation to become a predictive tool in equipment and process design.

The present task in CVD simulation includes the stabilization of the numerical codes to achieve fast turnaround time and the improvement of transport and chemical models to proceed from qualitative studies to quantitative predictions.

We will discuss models for heat and mass transport in low density gas mixtures and for homogeneous and heterogeneous reactions for a number of relevant CVD processes. Actual applications to problems in a submicron manufacturing environment will illustrate the presentation.
\end{abstract}

\section{Introduction}

In recent years numerical modelling of the processes that determine the chemical and physical behaviour of CVD equipment has gained increasing attention. Already more than a decade ago the basic work concerning the differential equations and the respective boundary conditions has been established [1] and a number of basic investigations for CVD processes have been published in the meantime.

Equipment simulation of reactors for undoped polysilicon deposition from $\mathrm{SiH}_{4}$ gas appears to be the most widely studied $[2,3,4]$, and the models have been validated through comparison with experiments [5]. CVD of silicon dioxide [6], silicon nitride [7], MOCVD for GaAs deposition [8], and tungsten CVD [9, 10,11, 12], have also been studied through simulation, although the chemical reaction models used have been less thoroughly investigated.

In general, most of these works seem to study the principal behaviour of each process, but few proceed as far as to the optimization of a particular piece of equipment. Through the availability of increased computing power it now becomes possible to use the simulation 
as a predictive tool in the design and optimization of equipment and processes which are used in industrial CVD applications. [13, 14].

In this development, which has been taking place recently three main tasks can be identified:

- Models and parameters for the chemical and physical models built into the programs must be improved to proceed from qualitative simulations to quantitative predictions. We will review some important features of the models in Section 2 of this paper.

- Stability and user friendliness of the computer programs must be enhanced to achieve fast turnaround time and enable simulation support in parallel to reactor and process development. We will review the current status of this task in Section 3.

- Pilot projects must be performed and documented to prove the agreement of the predictions with the finally manufactured reactors. Section 4 will present a number of examples from our work in the field of semiconductor technology to illustrate the way in which simulation of CVD can be efficiently used in industry.

\section{Model equations}

\subsection{Differential equations}

The gas flow in the reactor is simulated by the numerical solution of the mass-, chemicalspecies-, momentum-, and energy-continuity equations [15]. The mass continuity equation reads

$$
\frac{\partial \rho}{\partial t}+\nabla \cdot(\rho \mathbf{u})=0
$$

where $\rho$ is the density of the mixture, $t$ is time, and $u$ is the velocity vector.

Momentum continuity is described by the Navier-Stokes equation as

$$
\frac{\partial}{\partial t}\left(\rho u_{i}\right)+\nabla \cdot\left(\rho \mathbf{u} u_{i}\right)=\nabla \cdot\left(\mu_{m} \nabla u_{i}\right)-\frac{\partial}{\partial x_{i}} P+C_{i}
$$

where $i=1,2,3$ in a $3-\mathrm{D}$ geometry, $\mu_{m}$ is viscosity of the mixture, $P$ is pressure, and $C_{i}$ represents viscous terms in addition to those expressed by $\mu_{m}$.

The temperature can be calculated from the energy conservation equation

$$
\frac{\partial}{\partial t}(\rho H)+\nabla \cdot(\rho \mathbf{u} H)=\nabla \cdot\left(\kappa_{m} \nabla T\right)+S_{h}
$$

where $H$ is specific enthalpy that is related to the temperature through heat capacity $C_{p}$ by $d H=C_{p} d T, \kappa_{m}$ is thermal conductivity of the mixture, $T$ is temperature, and $S_{h}$ is the rate of heat generation per volume.

The conservation of chemical species considers the effect of both convection and diffusion for every particle ' $i$ ' in the reactor as

$$
\frac{\partial}{\partial t}\left(\rho m_{i}\right)+\nabla \cdot\left(\rho \mathbf{u} m_{i}\right)=\nabla \cdot \mathbf{j}_{i}+G_{i}
$$


where $m_{i}$ is mass fraction and $j_{i}$ is the mass flux of species ' $i$ ', and $G_{i}$ is the generation term in the bulk. Following Kleijn [4] we split the flux into ordinary diffusion, $\mathbf{j}_{i}^{C}$, and thermodiffusion, $\mathbf{j}_{i}^{T}$.

$$
\mathbf{j}_{i}=\mathbf{j}_{i}^{C}+\mathbf{j}_{i}^{T}
$$

The diffusion flux in a multicomponent mixture depends on the gradients of all the species present in the mixture and is governed by the Stefan-Maxwell relations, which in terms of mass fractions read [4]

$$
\nabla m_{i}+m_{i} \nabla(\ln M)=\sum_{j} \frac{M}{\rho M_{j} D_{i j}}\left(m_{i j} j_{j}^{C}-m_{j} \mathbf{j}_{i}^{C}\right)
$$

with $D_{i j}$ being the binary diffusion coefficient of species i in species $\mathrm{j}, M_{i}$ the molecular weight of species $i$ and $M$ the average molecular weight of the mixture

$$
M=\sum_{i} x_{i} M_{i}
$$

The thermodiffusion flux is given by

$$
\mathbf{j}_{i}^{T}=-D_{i}^{T} \nabla(\ln T)
$$

where $D_{i}^{T}$ is the thermodiffusion coefficient.

The ideal gas law $P=\rho R T / M$ is included in the equations to express the correct relation between density, pressure, and temperature.

\subsection{Transport parameters}

In the differential equations we have a number of transport parameters like the viscosity $\mu$, thermal conductivity $\kappa$, and diffusivities $D_{i j}$. It is clear that reliable simulation results require a correct description of these parameters as a function of temperature, pressure and mixture composition. Unfortunately there are not enough measured data available, especially for the more exotic gases used in many CVD processes.

Nevertheless, most of the necessary data can be derived from gas kinetic considerations estblished by Chapman and Enskog at the beginning of this century. In this theory all the transport parameters and also most of the equilibrium data of an ideal gas mixture are derived from the intermolecular potential distribution. The potential is approximated by the following function

$$
\phi(R)=\frac{4}{\epsilon}\left[(\sigma / R)^{12}-(\sigma / R)^{6}\right]
$$

where $\mathrm{R}$ is the distance from the molecule center. The two parameters $\sigma$ and $\epsilon$ characterize the specific kind of molecule and represent the collision diameter and the minimum energy of interaction between two molecular partners, respectively.

From the two parameters $\sigma$ and $\epsilon$ essentially all the important transport parameters can be calculated, except the heat capacity $c_{p}$. So, if we have measured values for the heat capacity $c_{p}$ and any other data (e.g. viscosity of the pure components or critical volume and pressure), that allow the deduction of the molecular parameters $\sigma$ and $\epsilon$ for each molecule, we can derive all the other transport parameters using the Chapman Enskog theory. 
Table 1: Coefficients used for the transport parameters. The heat capacity $C_{p}$ (in $[J /(\mathrm{mol}$. $K)]$ ) is given by the polynomial $C_{p}=A+B T+C T^{2}+D T^{3}$. The values for $W F_{6}, W F_{5}$, and $T E O S$ have been estimated from similar molecules.

\begin{tabular}{|l|ccccccc|}
\hline & $M_{i}$ & $\sigma_{i}$ & $\epsilon_{i} / k$ & $A$ & $B$ & $C$ & $D$ \\
\hline$H_{2}$ & $2 \mathrm{~kg} / \mathrm{kMol}$ & $2.827 \AA$ & $59.7 \mathrm{~K}$ & 27.1 & $9.3 \mathrm{E}-3$ & $-1.38 \mathrm{E}-5$ & $7.7 \mathrm{E}-9$ \\
$W F_{6}$ & $298 \mathrm{~kg} / \mathrm{kMol}$ & $5.21 \AA$ & $338 \mathrm{~K}$ & -28.2 & $7.0 \mathrm{E}-1$ & $-7.8 \mathrm{E}-4$ & $3.0 \mathrm{E}-7$ \\
$W F_{5}$ & $279 \mathrm{~kg} / \mathrm{kMol}$ & $5.21 \AA$ & $338 \mathrm{~K}$ & -28.2 & $7.0 \mathrm{E}-1$ & $-7.8 \mathrm{E}-4$ & $3.0 \mathrm{E}-7$ \\
$S_{i} F_{4}$ & $104 \mathrm{~kg} / \mathrm{kMol}$ & $4.88 \AA$ & $172 \mathrm{~K}$ & 26.8 & $2.2 \mathrm{E}-1$ & $-2.2 \mathrm{E}-4$ & $8.0 \mathrm{E}-8$ \\
$S_{i} H_{4}$ & $32 \mathrm{~kg} / \mathrm{kMol}$ & $4.08 \AA$ & $208 \mathrm{~K}$ & 11.2 & $1.2 \mathrm{E}-1$ & $-5.5 \mathrm{E}-5$ & $6.8 \mathrm{E}-9$ \\
$B_{2} H_{6}$ & $27.7 \mathrm{~kg} / \mathrm{kMol}$ & $4.70 \AA$ & $230 \mathrm{~K}$ & 5.4 & $1.8 \mathrm{E}-1$ & $-6.9 \mathrm{E}-5$ & $8.7 \mathrm{E}-9$ \\
$P H_{3}$ & $34 \mathrm{~kg} / \mathrm{kMol}$ & $3.98 \AA$ & $251 \mathrm{~K}$ & 23.2 & $4.4 \mathrm{e}-2$ & $1.3 \mathrm{E}-5$ & $-1.6 \mathrm{E}-8$ \\
$T E O S$ & $208 \mathrm{~kg} / \mathrm{kMol}$ & $6.90 \AA$ & $484 \mathrm{~K}$ & -54.9 & 1.1 & $-7.6 \mathrm{E}-4$ & $2.16 \mathrm{E}-7$ \\
$A r$ & $40 \mathrm{~kg} / \mathrm{kMol}$ & $3.54 \AA$ & $93.3 \mathrm{~K}$ & 20.8 & 0.0 & 0.0 & 0.0 \\
$H F$ & $20 \mathrm{~kg} / \mathrm{kMol}$ & $3.15 \AA$ & $330 \mathrm{~K}$ & 29.0 & $6.6 \mathrm{E}-4$ & $-2.0 \mathrm{E}-6$ & $2.5 \mathrm{E}-9$ \\
\hline
\end{tabular}

A variety of helpful formulae for this purpose and their accuracy are discussed in [16]. A consistent set, which we have used in our work is given in [12] and [5]. In Table 1 the values for $\sigma$ and $\epsilon$ and $c_{p}$ are tabulated for some of the gases used in CVD processes.

A special problem is given by the thermodiffusion coefficient $D_{i}^{T}$. There are very few if any measurements of the thermodiffusion coefficient in gas mixtures. So there is no experience with simplified approximations and their accuracy. A number of authors $[2,3,11]$ therefore go to the most rigorous formulae available in the literature $[17,18]$, hoping that this will yield the highest possible accuracy.

Unfortunately, those rigorous formulae look tremendously complicated and require a significant portion of additional computer time. We know of no results which prove that this effort is really necessary. Much simpler formulae are given by Wahl [19] and Kuo [20] which require considerably less coding and computational effort. However, these authors do not provide definite statements on the accuracy of their thermodiffusion coefficients.

\subsection{Chemical Reactions}

While the transport physics of CVD systems is based on a well established theory and on a reliable data base, the chemistry of the processes leaves many more open questions.

$\mathrm{SiH}_{4}$ based systems for undoped polysilicon deposition have been studied most intensively including as many as 16 intermediate species and 27 reactions. For this system quite a clear understanding has been developed, and the most important species and reactions have been identified. $[2,3,4]$. Similar studies, but with a smaller number of species have been performed also for $W F_{6}$ [23], TEOS chemistry [6], silicon nitride [7], and silicon carbide deposition [21].

In practical simulations it is not possible to use these detailed chemistry models in a 3-D reactor simulation. A viable solution is to reduce the complex chemistry to a very simple model for the deposition rate, which neglects all chemical species with low concentration and 
combines the high number of reactions into one phenomenological model.

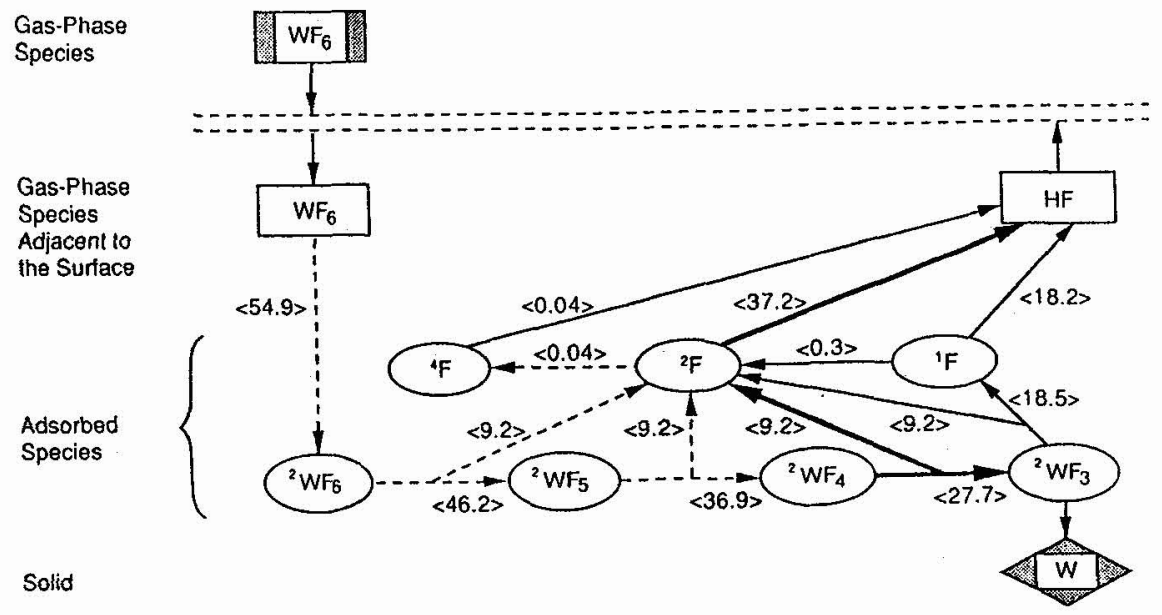

Figure 1: Major reaction pathways for CVD of tungsten (after [23]).

A typical example is the deposition of tungsten which occurs by reduction of tungsten hexafluoride through the overall reaction

$$
W F_{6}(g)+3 H_{2}(g) \longrightarrow W(s)+6 H F(g) .
$$

The deposition rate $R_{w}$ can be phenomenologically described by the following formula, which takes care of the mass-transfer and reaction-kinetics limited regimes

$$
\begin{gathered}
\frac{1}{R_{w}}=\frac{1}{R_{d}}+\frac{1}{R_{k}} \\
R_{d}=\frac{\rho}{\rho_{w}} D_{W F_{6}} \frac{m_{W F_{6}}}{\delta} \\
R_{k}=k_{o} \exp \left(\frac{-E_{a}}{k T}\right) P_{H_{2}}^{1 / 2}
\end{gathered}
$$

where $\rho$ and $\rho_{w}$ are the densities of the gas mixture and of CVD tungsten, respectively, $D_{W F_{6}}$ is the diffusion coefficient of $W F_{6}$ at the operating conditions of the first grid cell centre, and $\delta$ is the distance from the cell centre to the wafer, $k_{0}=4.08 \times 10^{6}\left(\mathrm{~nm} \mathrm{~min}^{-1} \mathrm{~Pa}^{-1 / 2}\right)$; and the activation energy $E_{a}=0.75 \mathrm{eV}$.

For a very fast chemical reaction $\left(R_{k} \gg 1\right)$ we are in the mass-transfer limited regime and the depostion rate approaches the maximum diffusive flux to the wafer surface. The kinetic limited regime is described by the Arrhenius equation as proportional to the square root of the partial pressure of $H_{2}$ [22]. The dependence of the deposition rate on $W F_{6}$ concentration enters only through the mass transport limitation, while the chemical reaction is taken as order zero in $W F_{6}$. This formula was first proposed by Ulacia [9] and has been proven to reproduce experimental data over a wide range of $W F_{6}$ partial pressures $[14,11]$.

A deeper investigation of this process has been performed by Arora and Pollard [23], who identified the dominant reaction pathways and derived a more complicated formula for 
the reaction rate as a function of temperature and concentrations. In Figure 1 we show a schematic of the chemistry investigated in this model. We see that the process in reality proceeds over several steps involving $W F_{5}, W F_{4}, W F_{3}$ radicals, which need not be considered in the simple formula (10).

However, when we do selective tungsten deposition the main reaction from $W F_{6}$ is blocked on insulating surfaces and only takes place on metallic surfaces. Nevertheless, a side path containing $W F_{5}$ still can lead to nucleation of tungsten on the insulating surfaces. Thus, if we want to study the selectivity of such a process we also have to take care of the $W F_{5}$ species, despite its very low concentration [12].

Comparable phenomenalogical deposition rate formulae have also been given for polysilicon [5], silicon nitride [7], and silicon oxide deposition [6].

\section{Numerical programs}

To solve the partial differential equations (1-4) on a computer the reactor geometry must be discretized by a large number of gridpoints and the continuous functions for pressure, density, temperature, and mass fractions must be approximated by their values on the grid nodes. From special assumptions about the functional behaviour of the functions between the grid nodes (constant, linear, etc.) we can solve the equations locally in a grid element and thus transfer the differential equations into a large system of algebraic equations, which correlate the values on neighbouring grid nodes. This large system must be linearized and then solved on the computer.

We can identify two main streams of programs with the basic difference in the structure of the grid. On the one hand we have structured grids, which can be derived by transformation from a regular rectangular grid, as is shown in Figure 2. These grids need not keep the orthogonal angle between the gridlines, but they maintain the regular topological structure, so that each point always has 4 neighbours in a plane and 6 neighbours in 3-D. Moreover every gridline can be followed through the whole simulation domain, but it need not be a straight line.

The regular structure makes programming and calculation easier, but usually requires more gridpoints. This is clear from Figure 2, since the regularity of the grid often requires many more gridpoints than it would be necessary to resolve the variations of the solution. Numerical methods used to solve the equations on structured grids are the finite difference (FD) and the finite volume (FV) methods [24].

If we use unstructured grids we can save a large number of grid points by terminating the grid lines in regions where the variation of the solution is expected to be small, as can be observed in Figure 2. However, since we loose the regularity of the grid, a lot of bookkeeping overhead is required in the program, to keep track of all the neighbours of a point. The numerical method used in unstructured grids is the Finite Element Method (FEM), but some new efforts have been reported, which try to extend the FV method also to unstructured grids [26].

So far structured grids seem to produce results with considerably less computational effort than unstructured grids. Calculation times for typical 3-D applications are several CPU hours to produce reasonable solutions. For programs using the FV method on structured grids a minicomputer or a large workstation is sufficient, while the FEM programs with unstructured grids need a large mainframe or a vector computer. 


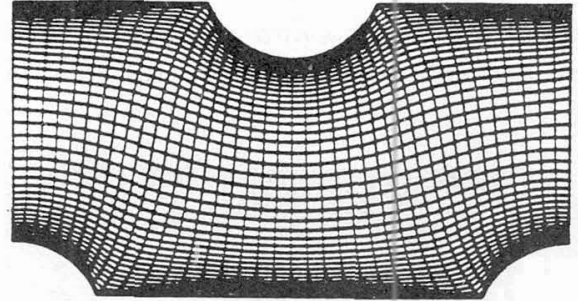

Structured

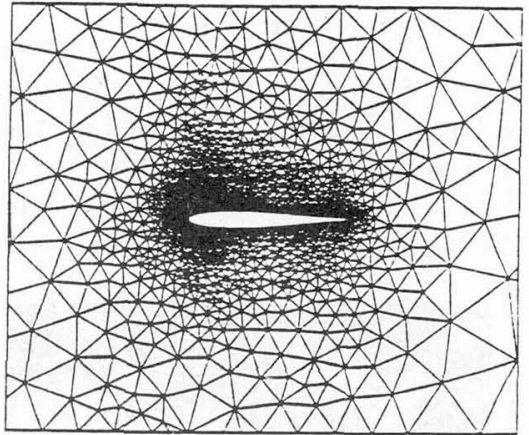

Unstructured

Figure 2: Different grid types for a fluid flow simulation (from [25]).

The computer codes used to solve practical problems can be split into two groups of rather different applications. In the academic world each group produces its own code, which is well adapted to the specific problem to be investigated and to the available hardware. However, usually the programs are not stable enough to be used by a different person, for a different problem, and on a different computer. We would estimate, that over $90 \%$ of the published results on CVD simulation were achieved with this type of code.

Another group of programs is mainly preferred by industry groups. These are derived from general purpose fluid flow programs $[27,28,29]$ produced by professional software houses and then modified to include special CVD models as described in Section 2 of this paper. In some cases these models are also offered for sale as special CVD versions of the programs. This is a very new development and stable programs have become available as recently as 1991. The advantage of these programs for the user is a higher support and user friendliness concerning at least the general features, which are not specific to CVD iInput menu, plotting routines, implementation support, etc). Nevertheless the problem is complicated enough to require an experienced specialist for an efficient use.

\section{Applications}

It is not the purpose of this paper to provide an excessive review of all the published applications of CVD simulation programs. For this purpose the recent excellent review of Jensen et al. [30] should be consulted. In this paper we will rather show a variety of examples from the industrial application, which we had to solve in a manufacturing environment for submicron silicon devices.

In all the examples the fluid flow simulator PHOENICS [27] was utilised to solve the transport equations (1)-(4). Appropriate subroutines were added to describe surface chemistry, thermal diffusion, and multicomponent diffusion according to equations $(10),(6)$, and (8). 


\subsection{Tungsten CVD}

In Figure 3 we show the gas flow and the temperature distribution in a cold wall, single wafer, tungsten CVD reactor [9] based on the reduction of tungsten hexafluoride by hydrogen.

In the reactor simulated, the wafer faces down onto the gas flow and is heated from the rear with halogen lamps. The gas is introduced at the bottom of the reactor and extracted at the top through four pumping ports.

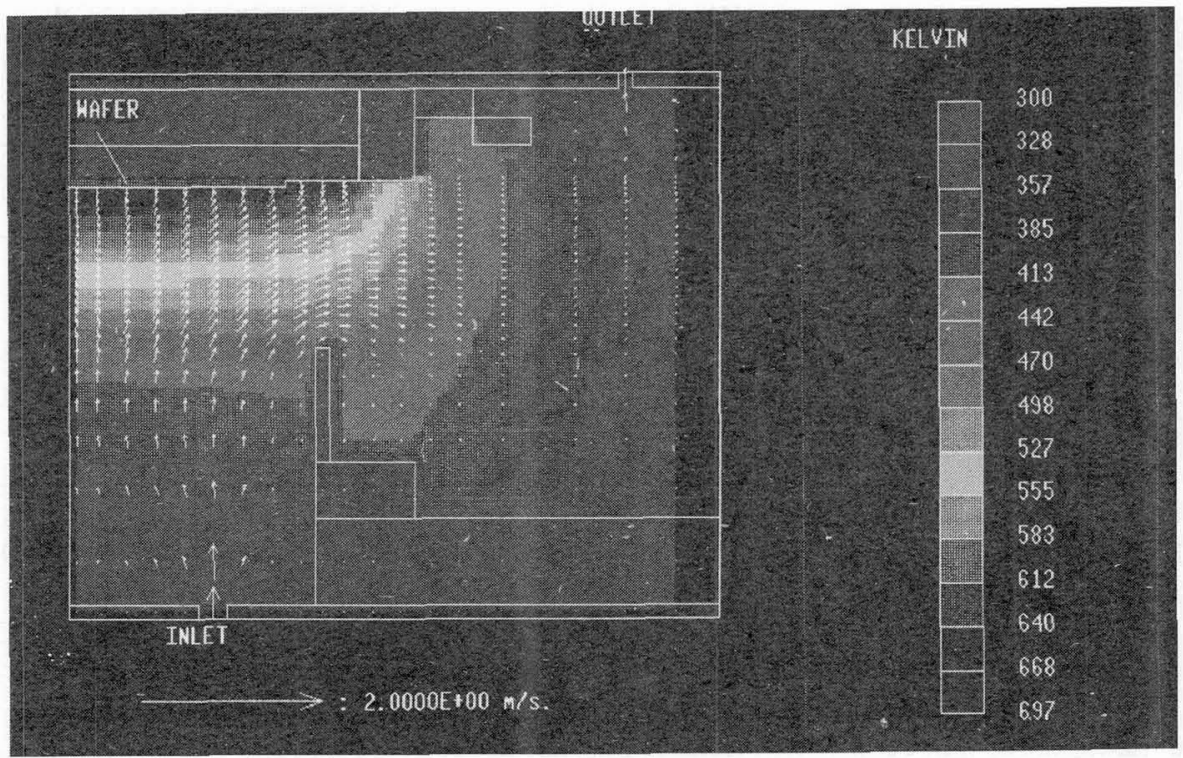

Figure 3: Velocity of gas flow and temperature contours inside the CVD reactor. The temperature increases as the gas approaches the hot wafer.

A typical application for reactor optimization is shown in Figure 4, where we plot the tungsten deposition rate along the wafer radius for two different heating arrangements. The upper line shows results for spatially uniform input radiation at the backside of a graphite wafer holder in close contact with the wafer. Because the heat loss at the outer holder edge is high, lower temperature and hence reduced deposition rates near the wafer periphery are found. The deposition uniformity is improved by the use of a non-uniform heating arrangement where the center of the wafer holder is screened from the incoming radiation, so that the temperature drop at the periphery could be partially compensated. This figure also illustrates simulated deposition results for a holder which was fully or partially screened from the input radiation. If total isolation is furnished, a possible improvement in homogeneity of more than a factor three is obtained as compared to the uniform heating arrangement. The experimental screening results indicate that about half of the incoming radiation is actually masked and further improvement should be possible with better screening. 


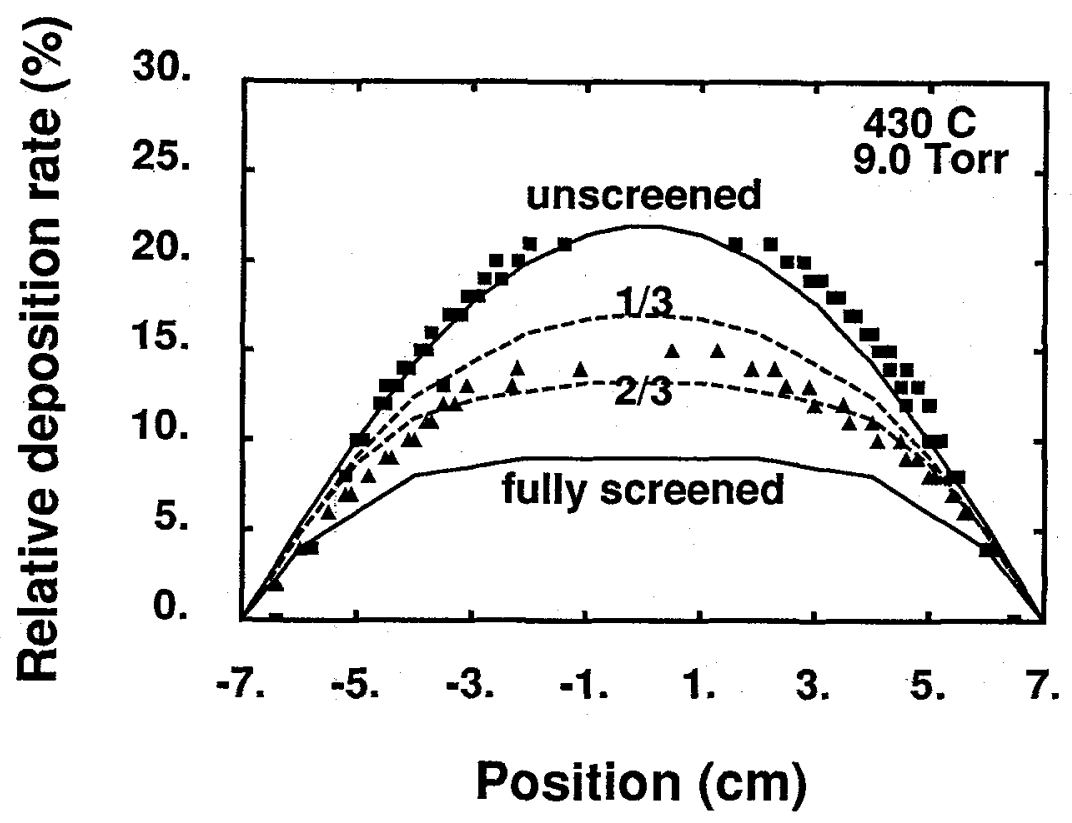

Figure 4: Deposition rate uniformity for a tungsten cold-wall CVD reactor. Inhomogeneous heating improves the uniformity compared to uniform heating.

\subsection{Thermodiffusion}

The effect of thermodiffusion is most relevant when heavy molecules diffuse in a light carrier gas under large temperature gradients. In Figure 5 and 6 we compare $W F_{6}$ concentrations in a $H_{2}$ carrier gas of the tungsten reactor described above both with and without the consideration of thermodiffusion, respectively. By thermodiffusion, the concentration values of the heavy molecules $W F_{6}$ are enhanced in the cold regions of the reactor and reduced near the hot wafer. This effect can significantly reduce the reaction rate. Figure 7 shows a concentration profile vertical to the wafer plane both with and without thermodiffusion. We find a characteristic maximum near the inlet and a factor of 2 reduction at the surface. Thermodiffusion effects are considerably smaller, when the molecular weight of reacting species and carrier gas are similar (e.g. $\mathrm{SiH}_{4}$ in $\mathrm{Ar}$ ).

\subsection{Selective Tungsten Deposition}

In this simulation we have calculated concentration contours of the intermediate species $W F_{5}$, which is suspected to be responsible for selectivity loss as described in Section 2 .

The deposition is performed in the same reactor as before, but now only $1 \%$ of the wafer surface is reactive for the $W F_{6}$ reduction process. However, the wafer is surrounded by a susceptor, which is covered with tungsten from earlier depositions and is therefore $100 \%$ reactive. During the $W F_{6}$ reduction at the reactive surfaces also a small amount of $W F_{5}$ is produced and desorbs into the gas volume. Since $W F_{5}$ can trigger an unwanted tungsten nucleation on the passive surfaces it is important to know its concentration at the wafer and to minimize it for improved selectivity. 


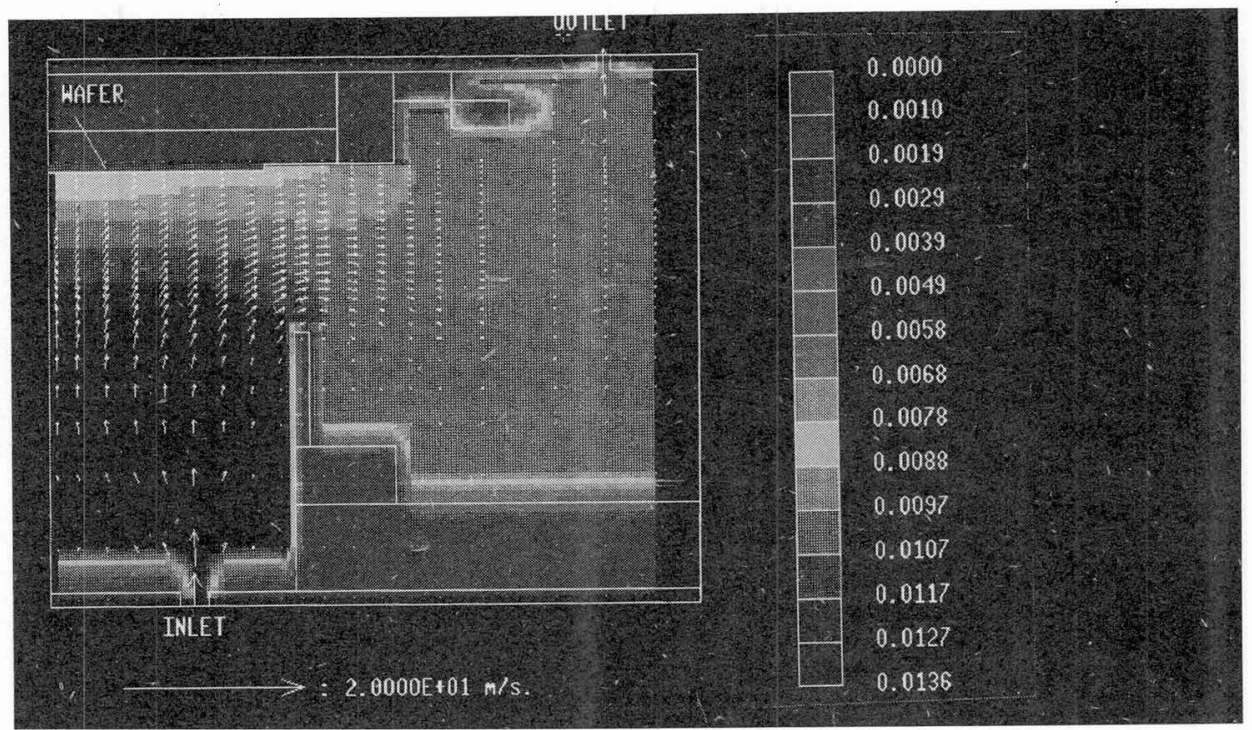

Figure 5: Concentration contours of tungsten hexafluoride inside the reactor without consideration of the thermodiffusion effect.

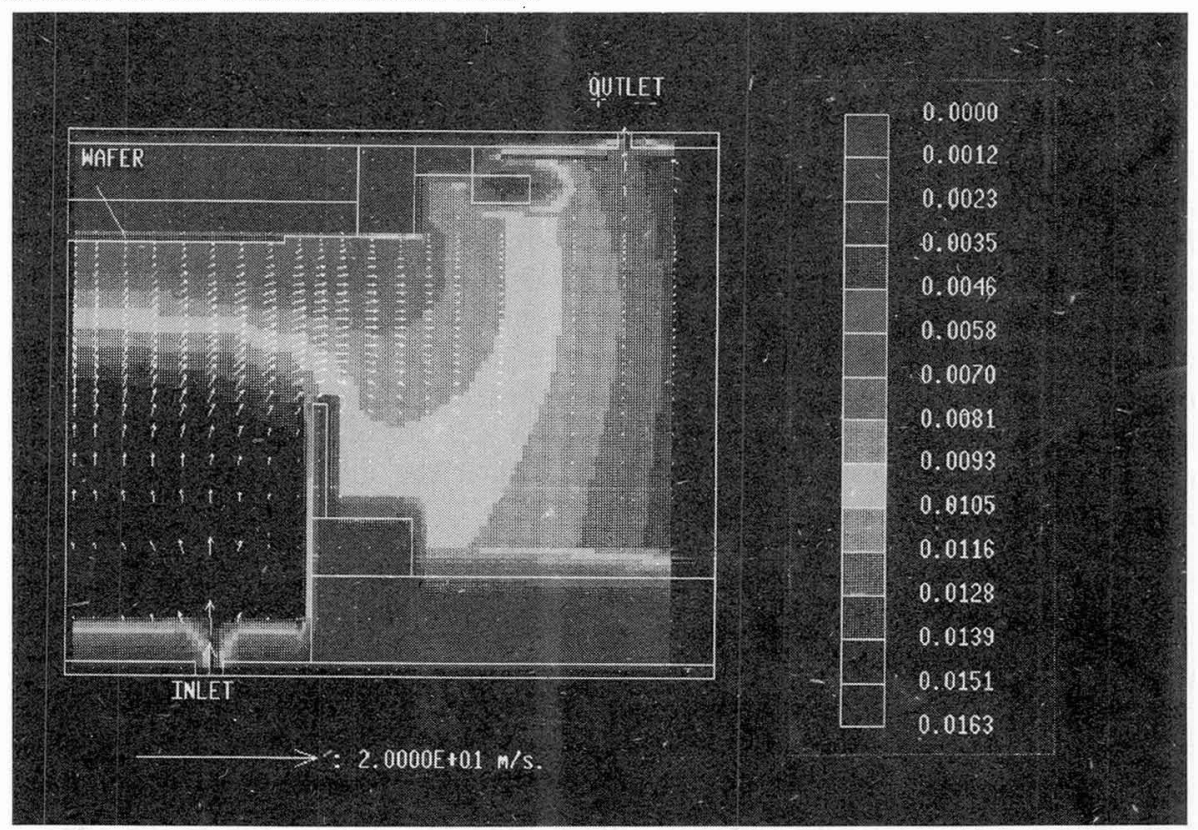

Figure 6: Concentration contours of tungsten hexafluoride inside the reactor with consideration of the thermodiffusion effect. 


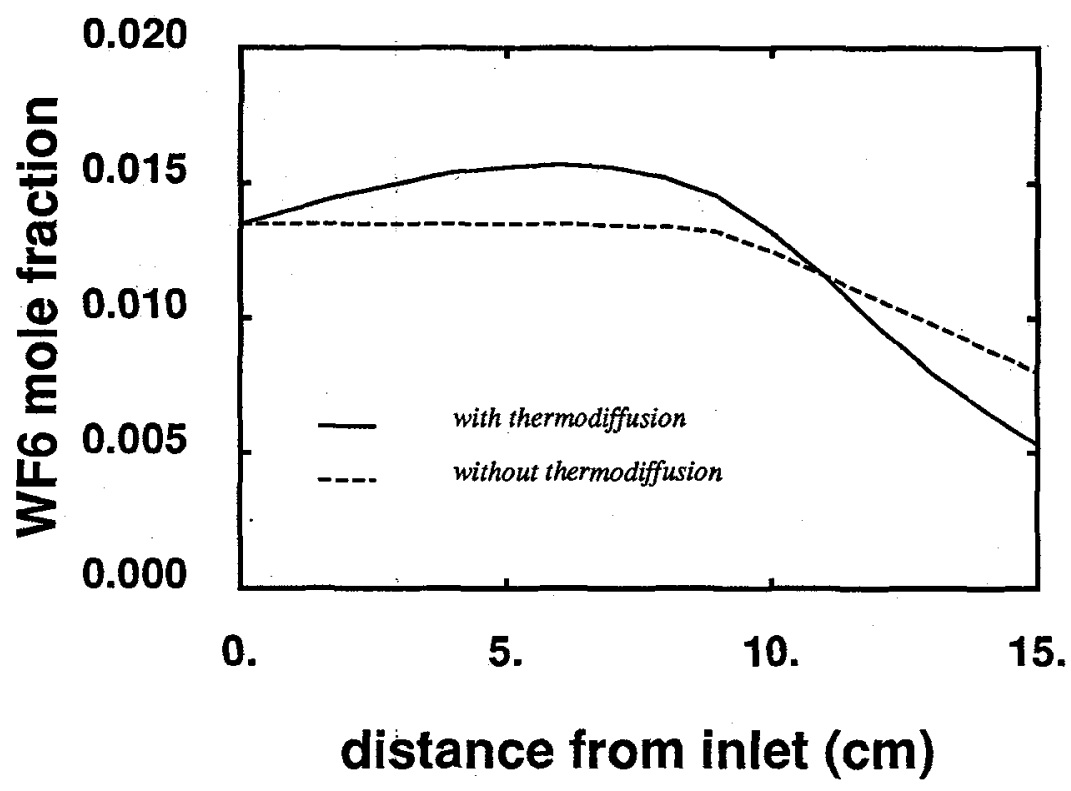

Figure 7: concentration profile vertical to the wafer plane both with and without thermodiffusion

In Figure 8 we give the concentration of the byproduct $W F_{5}$, which has a maximum at the reactive susceptor and shows a drop in excess of a factor of 10 between the edge and the center of the wafer. This leads to enhanced tungsten nucleation and selectivity loss at the periphery of the wafer [12].

Figure 9 illustrates the influence of a modified reactor design, where the outlet gap at the wafer edge was reduced from $60 \mathrm{~mm}$ to $6 \mathrm{~mm}$. This causes an increased gas speed near the hot susceptor which drives the byproduct $W F_{x}$ away from the wafer. Therefore the concentration near the wafer is reduced by a factor of $2-3$ compared with the original design.

However, it is well known that besides this geometric optimization, there is also a strong influence from surface cleanliness and wafer history on selectivity.

\section{Discussion}

Based on the results illustrated here, it is possible to obtain a large leverage from numerical simulation as a process and design tool. From our experience some of the most important advantages of equipment simulation at the fluid dynamic level, for both equipment manufacturers and semiconductor companies, can be summarized as follows:

- Simulation provides a visual output on the contours of pressure, temperature, gas velocity, and concentration of different chemical species as a function of position, which helps the understanding of the process.

- It is possible to compute self-consistently the uniformity of deposition based on the local values of temperature and partial pressures of the different species, which might 


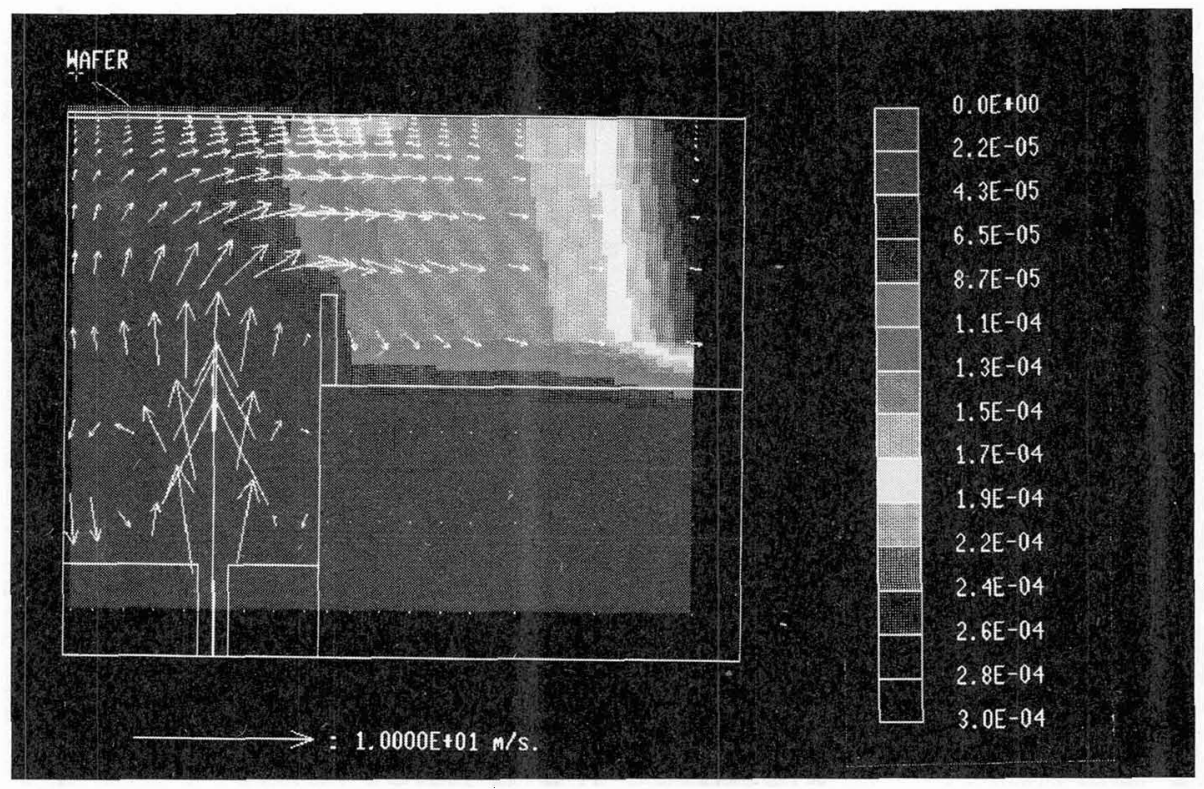

Figure 8: Concentration contours of $W F_{5}$ inside the reactor. $W F_{5}$ desorbs from the hot susceptor as a byproduct of the $W F_{6}$ reduction and is adsorbed at the wafer surface.

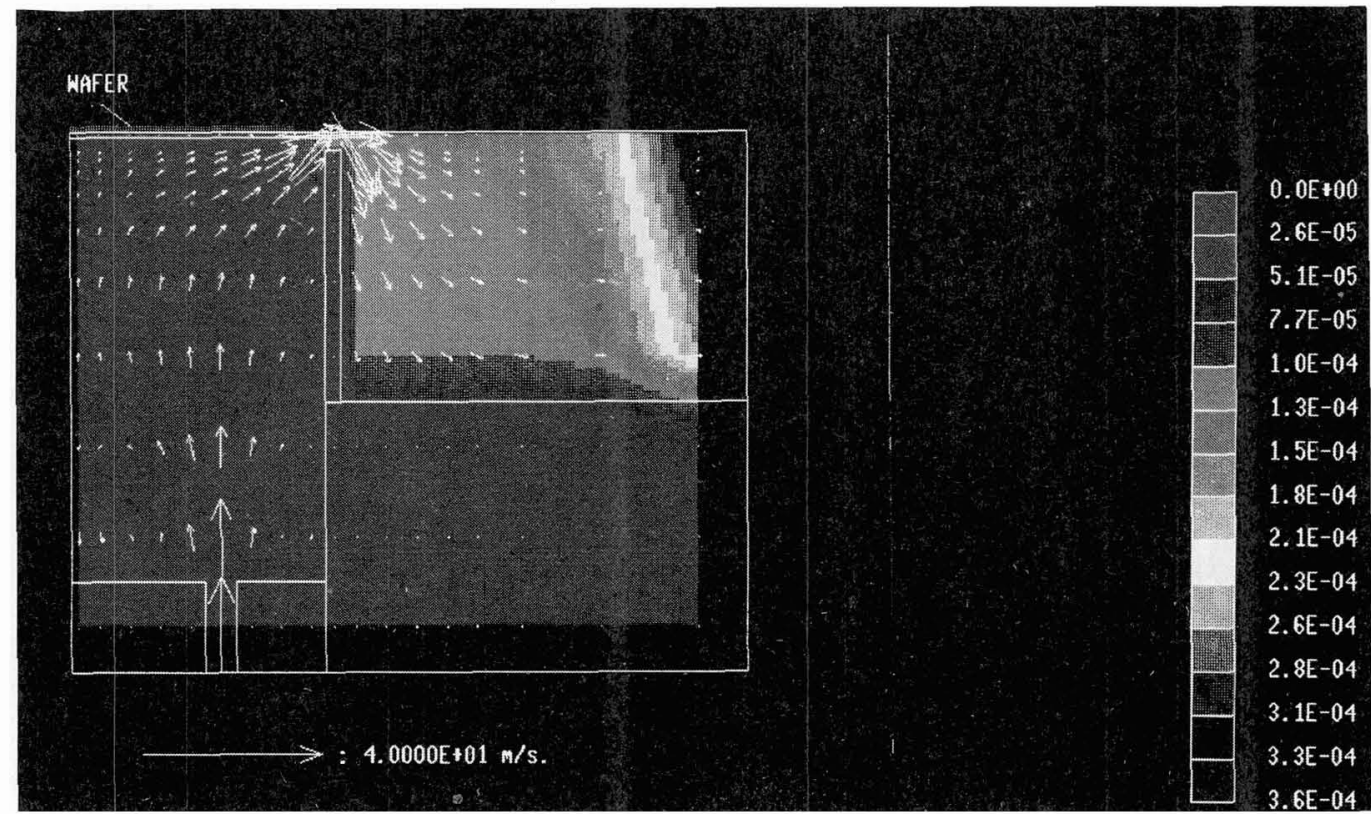

Figure 9: Gas velocity and Concentration contours of $W F_{5}$ for a reduced outlet gap of $6 \mathrm{~mm}$. The small gap increases the gas velocity near the susceptor compared to figure 3 . 


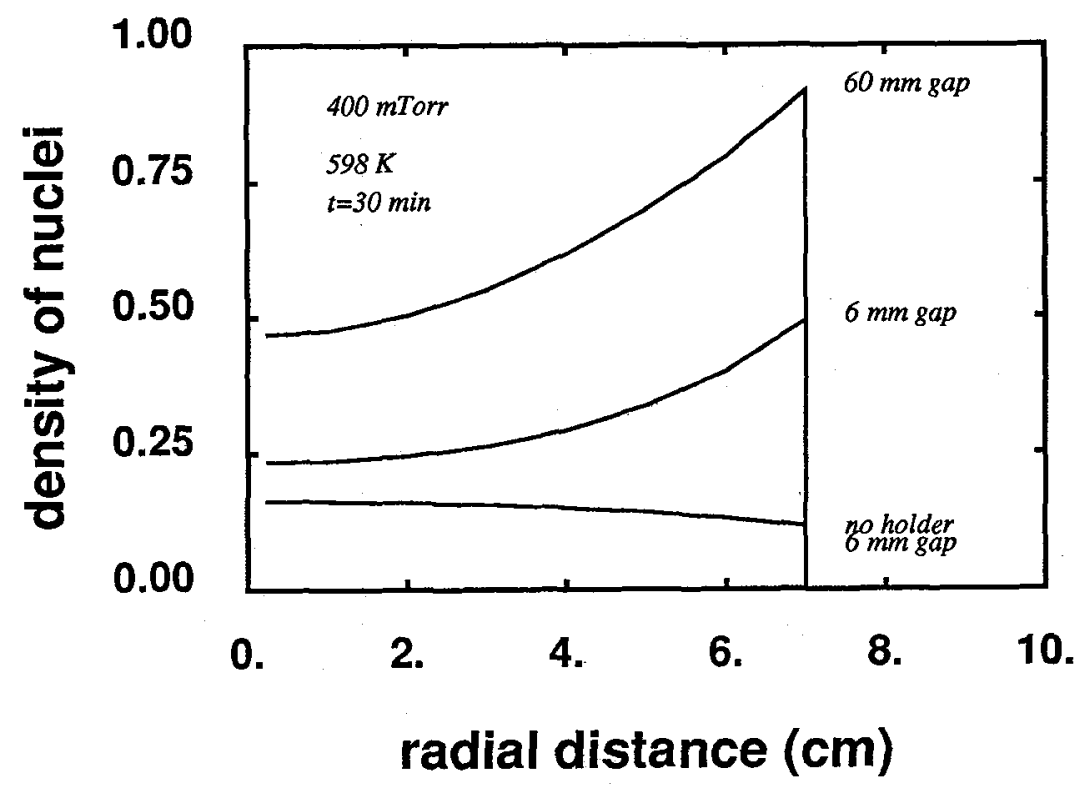

Figure 10: Density of tungsten nuclei on the nonreactive surface as a function of radial distance on the wafer. Three different reactor designs are compared.

be quite different from the user-specified input conditions.

- This simulation tool allows the design engineer to investigate several hardware configurations to find the one that provides the best expected results, even before any piece of equipment has been machined.

- When there are processing or uniformity problems, this technique narrows the possible variables that may cause the undesired effects (i.e. recirculations, mass-transfer limitation, temperature, turbulence, inadequate gas flow). The numerical answer does not directly detect the problem, but rather indicates the variables that are not likely to cause it.

- Once there is an adequate model for the reactor, simulation lets the process and design engineer investigate the conditions of pressure, temperature, gas flow for higher deposition rates, and better uniformity, therefore improving yield and throughput.

- When good physical and chemical models have been developed, it is possible to investigate many different reactor geometries to determine the best reactor with a minimal effort. This has two main applications, the first relates to specific design of one equipment supplier (equipment design) and, the second, on the decision making of the consumer to determine the best machine on the market (process design).

- It is an open question whether this tool will reduce development costs; however, based on the arguments given above it is unquestionable that equipment produced based on these simulations will be much better optimized for any specific process. 
Although equipment simulation provides useful information, we must stay aware of the inherent limitations of the fluid-mechanical approach.

- All the calculated distributions are macroscopic quantities. That means they are averages over several mean free paths of the molecules in the gas (about $0.2 \mathrm{~mm}$ for 1 Torr and 600 degrees $C$ ). Therefore we cannot expect to obtain information about things like step coverage and deposition profiles, which are quantities on a much lower microscopic scale.

- If the pressure is reduced into the mTorr range, the mean free path becomes comparable to the reactor dimensions and we enter the Knudsen transport regime; here, corrections for the fluid-mechanical description ought to be included.

- Gas phase chemistry enters the equations only via generation and recombination terms, which require a priori knowledge of reaction kinetics data. In many cases these data are not available especially at high pressures or in highly energetic systems such as plasma enhanced CVD processes

- For the source of energy - either radiative or electromagnetic as in plasma processsing - very simple models are used so far, which rely mainly on experimentally determined values. Much more work is needed in this area to get predictive modelling tools also for energy source optimization

From this discussion, it is hoped that the reader will be able to identify the main advantages that numerical computation, in the form of equipment simulation, can contribute to advancement of state-of-the-art technology in CVD equipment and process development.

\section{Acknowledgements}

I would like to thank especially J. Ignacio Ulacia F., who contributed a significant amount to the work presented here, before he left SIEMENS to found his own consulting company QUALTEC. The contributions of S.Howell, Chr. Hopfmann, P. Flynn, and A. Kersch are also gratefully acknowledged. Moreover, many interesting discussions have been provided by engineers from the SIEMENS semiconductor processing line and also from the equipment companies responsible for the design of our CVD reactors.

\section{References}

[1] G. Wahl, Thin Solid Films 40 (1977) 13

[2] K.F Roenigk and K.F. Jensen, J. Electrochem. Soc. 132 (1985) 449

[3] M.E. Coltrin, R.J. Kee, and G.H. Evans J. Electrochem. Soc. 136 (1989) 819

[4] C.R. Kleijn, Th. H. van der Meer, and C.J. Hoogendoorn, J. Electrochem. Soc. 136 [11] (1989) 3423

[5] Ch. Hopfmann, J. Ignacio Ulacia F., and Ch. Werner, submitted to Appl. Surf. Sci. 
[6] S.R. Kalidindi and S.B. Desu, J. Electrochem. Soc. 137 (1990) 624.

[7] K.F Roenigk and K.F. Jensen, J. Electrochem. Soc. 134 (1987) 1777.

[8] H. Moffat and K.J. Jensen, Journal of Crystal Growth 77 (1986) 108

[9] J.I. Ulacia F., S. Howell, H. Körner, and Ch. Werner, Appl. Surf. Sci. 38 (1989) 370

[10] C.R. Kleijn, C.J. Hoogendoorn, A. Hasper, J. Holleman, and J. Middlehoek, Workshop of CVD tungsten, copper and other advanced materials for VLSI/ULSI applications, September (1989).

[11] C.R. Kleijn, C.J. Hoogendoorn, A. Hasper, J. Holleman, and J. Middlehoek, J. Electrochem. Soc. 138 (1991) 509.

[12] Chr. Werner, Ch. Hopfmann, J. Ignacio Ulacia F., and P. Flynn, submitted to J. Electrochem Society

[13] Chr. Werner, J.I. Ulacia F., and S. Howell, Symposium on VLSI technology, May 89 in Kyoto Japan, p. 49.

[14] J. Ignacio Ulacia F.,Christoph Werner, SOLID STATE TECHNOLOGY ,November 1990,p.107 and December 1990, p.71

[15] R.B. Bird, W.E. Stewart; E.N. Lightfoot, Transport Phenomena, John Wiley \& Sons New York 1960.

[16] R.C. Reid, J.M. Prausnitz, B.R. Poling The Properties of Gases and Liquids, Fourth Edition Mc Graw-Hill, New York 1987

[17] J.O. Hirschfelder, C.F. Curtiss, R.B. Bird, 'Molecular Theory of Gases and Liquids', John Wiley \& Sons New York 1954.

[18] G.Dixon-Lewis in COMBUSTION CHEMISTRY W. Gardiner (ed.), Springer N.Y. 1984 p. 21

[19] G. Wahl, in Proc. V th Euro CVD-Conf. on CVD, J.O. Carlsson (ed), (1985) p.88

[20] K. K. Kuo,Principles of Combustion, p.669, John Wiley \& Sons New York 1986

[21] M.D.Allendorf and R.J.Kee, Abstract 616, Electrochem. Soc. Fall Meeting, Seattle 1990.

[22] C.M. McConica and K. Krishnamani, J. Electrochem. Soc. 133 (1986) 2542.

[23] R. Arora and R. Pollard, J. Electrochem. Soc. 138 (1991) 1523

[24] S.V. Patankar, Numerical Heat Transfer and Fluid Flow, McGraw-Hill Book Co., New York (1980).

[25] Z. Sheikh,"FLUENT and Chemical Vapor deposition", SEMATECH workshop on Modelling for Equipment Design, Gaithersburgh, (1990)

[26] D.B. Spalding, "Phoenics New Features and Develpments", The German Phoenics User Conference, Baden Baden, (1990) 
[27] H.I. Rosten and D.B. Spalding, The PHOENICS Beginner's Guide, CHAM TR/100, Wimbledon, (1987).

[28] The FLUENT User Manual, Creare.x Inc., New Hampshire 1990.

[29] The FIDAP User Manual, Fluid Dynamics International, Evanston, Mlinois (1990).

[30] K.F.Jensen,E.O.Einset, and D.I.Fotiadis, Ann. Rev. Fluid Mech. 23 (1991) 197. 